Proceedings Book of International Work ume: 1 (2016)) June 2-4. 2016, Istanbl, Turkey. Editors: Nurettin Cenk Turgay, Elif Özkara Canfes, Joeri Van der Veken and Cornelia-Livia Bejan Recieved: February 8, 2017 DOI: 10.24064 /iwts2016.2017.7

\title{
On Pseudo-Umbilical Rotational Surfaces with Pointwise 1-Type Gauss Map in $\mathbb{E}_{2}^{4}$
}

\author{
Burcu Bektaş, Elif Özkara Canfes, Uğur Dursun
}

Burcu Bektaş: Istanbul Technical University, Faculty of Science and Letters, Department of Mathematics, 34469, Maslak, Istanbul, Turkey, e-mail:bektasbu@itu.edu.tr,

Elif Özkara Canfes: Istanbul Technical University, Faculty of Science and Letters, Department of Mathematics, 34469, Maslak, Istanbul, Turkey, e-mail:canfes@itu.edu.tr,

Uğur Dursun: Işık University, Faculty of Arts and Sciences, Department of Mathematics, 34980, Şile, Istanbul, Turkey, e-mail:ugur.dursun@isikun.edu.tr

\begin{abstract}
In this work, we study two families of rotational surfaces in the pseudo-Euclidean space $\mathbb{E}_{2}^{4}$ with profile curves lying in 2-dimensional planes. First, we obtain a classification of pseudo-umbilical spacelike surfaces and timelike surfaces in these families. Then, we show that in this classification there exists no a pseudo-umbilical rotational surface in $\mathbb{E}_{2}^{4}$ with pointwise 1type Gauss map of second kind. Finally, we determine such pseudo-umbilical rotational surfaces in $\mathbb{E}_{2}^{4}$ having pointwise 1-type Gauss map of first kind.

Keywords. Rotational Surfaces. Pseudo-Umbilical Surfaces . Gauss Map - Pointwise 1-Type Gauss Map.

MSC 2010 Classification. Primary: 53B25; Secondary:53C50.
\end{abstract}

\section{INTRODUCTION}

In late 1970, the theory of finite type submanifolds of Euclidean submanifolds was introduced by B.-Y. Chen, [7]. Since then, many mathematicians have characterized or classified submanifolds of Euclidean space or pseudo-Euclidean space in terms of finite type. Later, B.-Y.Chen and P. Piccinni extended the notion of finite type of submanifolds to Gauss map of submanifolds, [8]. The report [9] and the second edition of above mentioned book [10] are useful references to understand recent developments and open problems of this area.

A smooth map $\phi: M \longrightarrow \mathbb{E}_{s}^{m}$ from a (pseudo)-Riemannian manifold into a (pseudo)-Euclidean space is called of finite type if it has a finite spectral decomposition

$$
\phi=\phi_{0}+\sum_{i=1}^{k} \phi_{i}
$$


where $\phi_{0}$ is a constant map, and each non-constant maps $\phi_{i}$ satisfies $\Delta \phi_{i}=\lambda_{i} \phi_{i}$ for some constant $\lambda_{i} \in \mathbb{R}$. If the spectral decomposition (1.1) contains exactly $k$ terms with different values for $\lambda_{i}$, then the map $\phi$ is called of $k$-type. Thus, a (pseudo)-Riemannian submanifold $M$ of a (pseudo)-Euclidean space has 1type Gauss map $\nu$ if and only if $\Delta \nu=\lambda(\nu+C)$ for some $\lambda \in \mathbb{R}$ and for some constant vector $C$.

On the other hand, it was observed that the Gauss map of some submanifolds such as helicoid, catenoid, right cones in $\mathbb{E}^{3}$ and Enneper's hypersurfaces in $\mathbb{E}_{1}^{n+1}$ satisfies

$$
\Delta \nu=f(\nu+C)
$$

for some smooth function $f$ on $M$ and some constant vector $C,[13,16]$. This gives a new terminology, namely that, a submanifold of a (pseudo)-Euclidean space is said to have pointwise 1-type Gauss map if it satisfies (1.2). In particular, if $C$ is zero, it is said to be of the first kind. Otherwise, it is said to be of the second kind.

Also, rotational surfaces in a (pseudo)-Euclidean space which are the main focus of the present paper are another active research field in differential geometry. In 1919, C. L. Moore introduced generel rotational surfaces in the four dimensional Euclidean space, [19]. A rotational surface in $\mathbb{E}^{4}$ is a surface left invariant by a rotation in $\mathbb{E}^{4}$ which is defined as a linear transformation of positive determinant preserving distance and leaving one point fixed. Moreover, F. Cole studied the general theory of rotation in $\mathbb{E}^{4}$, [12].

The rotational surfaces in the pseudo-Euclidean space $\mathbb{E}_{2}^{4}$, called Vranceanu rotational surfaces which is a particular case of the rotational surfaces studied in this article were studied for different purposes. The complete classification of Vranceanu rotational surfaces in $\mathbb{E}_{2}^{4}$ with zero mean curvature was obtained in [15]. It was proved that a flat rotational surface in $\mathbb{E}_{2}^{4}$ with pointwise 1-type Gauss map is either the product of two plane hyperbolas or the product of a plane circle and a plane hyperbola, [17].

In [1], F. K. Aksoyak and Y. Yayl gave a classification of flat general rotational surfaces with pointwise 1-type Gauss map in the pseudo-Euclidean space $\mathbb{E}_{2}^{4}$ which includes similar results given in [17].

Recently, Y. Aleksieva, V. Milousheva and N. C. Turgay studied general rotational surfaces in the pseudo-Euclidean space $\mathbb{E}_{2}^{4}$ with zero mean curvature vector in [2] and then the first author, E. Canfes and U. Dursun classified such rotational surfaces with pointwise 1-type Gauss map in [4].

Moreover, there are many studies about the rotational surfaces in the pseudoEuclidean space and different spaces with pointwise 1-type Gauss map, [3, 11, $18]$.

On the other hand, pseudo-umbilical submanifolds are also well-known and have been studied in many articles, [6, 14, 5].

In this article, we consider two families of rotational surfaces in the pseudoEuclidean space $\mathbb{E}_{2}^{4}$ with profile curves lying in 2-dimensional planes. First, we determine the pseudo-umbilical rotational surfaces in these families. Then, we show that there exists no a non-planar pseudo-umbilical rotational surface in 
these families with pointwise 1-type Gauss map of the second kind. Finally, we give a classification of all such pseudo-umbilical surfaces in $\mathbb{E}_{2}^{4}$ with pointwise 1-type Gauss map of the first kind.

\section{$2 \quad$ PRELIMINARIES}

\subsection{Basics of Submanifold Theory}

Let $\mathbb{E}_{t}^{m}$ be the $m$-dimensional pseudo-Euclidean space with the canonical metric given by

$$
\tilde{g}=\sum_{i=1}^{m-t}\left(d x_{i}\right)^{2}-\sum_{i=m-t+1}^{m}\left(d x_{i}\right)^{2},
$$

where $\left(x_{1}, x_{2}, \ldots, x_{m}\right)$ is a standard rectangular coordinate system in $\mathbb{E}_{t}^{m}$.

For a point $\mathbf{x}_{\mathbf{0}} \in \mathbb{E}_{t}^{m}$ and $c \neq 0$, we put

$$
\begin{aligned}
& \mathbb{S}_{t}^{m-1}\left(\mathbf{x}_{\mathbf{0}}, c\right)=\left\{\mathbf{x} \in \mathbb{E}_{t}^{m} \mid\left\langle\mathbf{x}-\mathbf{x}_{\mathbf{0}}, \mathbf{x}-\mathbf{x}_{\mathbf{0}}\right\rangle=c^{-1}\right\} \text { if } c>0 \\
& \mathbb{H}_{t}^{m-1}\left(\mathbf{x}_{\mathbf{0}}, c\right)=\left\{\mathbf{x} \in \mathbb{E}_{t+1}^{m} \mid\left\langle\mathbf{x}-\mathbf{x}_{\mathbf{0}}, \mathbf{x}-\mathbf{x}_{\mathbf{0}}\right\rangle=c^{-1}\right\} \text { if } c<0
\end{aligned}
$$

where $\langle$,$\rangle denotes the indefinite inner product associated to \tilde{g}$. $\mathbb{S}_{t}^{m-1}\left(\mathbf{x}_{\mathbf{0}}, c\right)$ and $\mathbb{H}_{t}^{m-1}\left(\mathbf{x}_{\mathbf{0}}, c\right)$ are called, respectively, a pseudo-sphere and a pseudo-hyperbolic space. When $\mathbf{x}_{0}$ is the origin, we simply denote $\mathbb{S}_{t}^{m-1}(\mathbf{0}, c)$ and $\mathbb{H}_{t}^{m-1}(\mathbf{0}, c)$ by $\mathbb{S}_{t}^{m-1}(c)$ and $\mathbb{H}_{t}^{m-1}(c)$.

A vector $v \in \mathbb{E}_{t}^{m}$ is called spacelike (resp., timelike) if $\langle v, v\rangle>0$ or $v=0$ (resp., $\langle v, v\rangle<0$ ). A vector $v$ is called lightlike if it is non-zero and it satisfies $\langle v, v\rangle=0$.

From now on, we use the following convention on the range of indices:

$$
1 \leq A, B, C, \ldots \leq n+2, \quad 1 \leq i, j, k, \ldots \leq n, n+1 \leq r, s, t, \ldots \leq n+2 .
$$

Let $M$ be an oriented $n$-dimensional submanifold in an $(n+2)$-dimensional pseudo-Euclidean space $\mathbb{E}_{2}^{n+2}$. We denote the Levi-Civita connections of $\mathbb{E}_{2}^{n+2}$

and $M$ respectively, by $\widetilde{\nabla}$ and $\nabla$. Then, we choose an oriented local orthonormal frame $\left\{e_{1}, \ldots, e_{n+2}\right\}$ on $M$ with $\varepsilon_{A}=\left\langle e_{A}, e_{A}\right\rangle= \pm 1$ such that $e_{1}, \ldots, e_{n}$ are tangent to $M$ and $e_{n+1}, e_{n+2}$ are normal to $M$ in $\mathbb{E}_{2}^{n+2}$. Denote the dual frame and connection forms associated to $\left\{e_{1}, \ldots, e_{n+2}\right\}$ by $\left\{\omega^{1}, \ldots, \omega^{n+2}\right\}$ and $\omega_{A B}$, respectively.

The Gauss and Weingarten formulas are given, respectively, by

$$
\begin{aligned}
& \widetilde{\nabla}_{e_{k}} e_{i}=\sum_{j=1}^{n} \varepsilon_{j} \omega_{i j}\left(e_{k}\right) e_{j}+\sum_{r=n+1}^{n+2} \varepsilon_{r} h_{i k}^{r} e_{r}, \\
& \widetilde{\nabla}_{e_{k}} e_{r}=-A_{r}\left(e_{k}\right)+\sum_{s=n+1}^{n+2} \varepsilon_{s} \omega_{r s}\left(e_{k}\right) e_{s},
\end{aligned}
$$


where $h_{i j}^{r}$ is the coefficients of the second fundamental form $h$, and $A_{r}$ the Weingarten map in the direction $e_{r}$.

The mean curvature vector $H$ and the scalar curvature $S$ of $M$ in $\mathbb{E}_{2}^{n+2}$ are defined, respectively, by

$$
\begin{aligned}
H & =\frac{1}{n} \sum_{r=n+1}^{n+2} \varepsilon_{r} \operatorname{tr} A_{r} e_{r}, \\
S & =n^{2}\langle H, H\rangle-\|h\|^{2},
\end{aligned}
$$

where $\|h\|^{2}=\sum_{i, j=1}^{n} \sum_{r=n+1}^{n+2} \varepsilon_{i} \varepsilon_{j} \varepsilon_{r}\left(h_{i j}^{r}\right)^{2}$. A submanifold $M$ is called minimal if $H$ vanishes identically and a non-minimal submanifold is called pseudoumbilical if there exist a smooth function $\rho$ such that $A_{H}=\rho \mathrm{I}$, where $I$ is an identity $n \times n$ matrix and $\rho$ is a smooth function on $M$. In particular, the Gaussian curvature $K$ which is also defined by $K=\varepsilon_{3} \operatorname{det} A_{3}+\varepsilon_{4} \operatorname{det} A_{4}$ is half of the scalar curvature $S$ for $n=2$. If $K$ vanishes identically, the surface $M$ is called flat.

The Codazzi equations of $M$ in $\mathbb{E}_{2}^{n+2}$ are given by

$$
\begin{aligned}
& h_{i j, k}^{r}=h_{j k, i}^{r}, \\
& h_{j k, i}^{r}=e_{i}\left(h_{j k}^{r}\right)+\sum_{s=n+1}^{n+2} \varepsilon_{s} h_{j k}^{s} \omega_{s r}\left(e_{i}\right)-\sum_{\ell=1}^{n} \varepsilon_{\ell}\left(\omega_{j \ell}\left(e_{i}\right) h_{\ell k}^{r}+\omega_{k \ell}\left(e_{i}\right) h_{\ell j}^{r}\right) .
\end{aligned}
$$

Also, from the Ricci equation of $M$ in $\mathbb{E}_{2}^{n+2}$, we have

$$
R^{D}\left(e_{j}, e_{k} ; e_{r}, e_{s}\right)=\left\langle\left[A_{e_{r}}, A_{e_{s}}\right]\left(e_{j}\right), e_{k}\right\rangle=\sum_{i=1}^{n} \varepsilon_{i}\left(h_{i k}^{r} h_{i j}^{s}-h_{i j}^{r} h_{i k}^{s}\right)
$$

where $R^{D}$ is the normal curvature tensor.

The gradient of a smooth function $f$ on $M$ is defined by $\nabla f=\sum_{i=1}^{n} \varepsilon_{i} e_{i}(f) e_{i}$, and the Laplace operator acting on $M$ is $\Delta=\sum_{i=1}^{n} \varepsilon_{i}\left(\nabla_{e_{i}} e_{i}-e_{i} e_{i}\right)$.

\subsection{Gauss Map}

Let $G(m-n, m)$ be the Grassmannian manifold consisting of all oriented ( $m-$ $n$ )-planes through the origin of a pseudo-Euclidean space $\mathbb{E}_{t}^{m}$ with index $t$, and let $\bigwedge^{m-n} \mathbb{E}_{t}^{m}$ be the vector space obtained by the exterior product of $m-$ $n$ vectors in $\mathbb{E}_{t}^{m}$. Let $f_{i_{1}} \wedge \cdots \wedge f_{i_{m-n}}$ and $g_{i_{1}} \wedge \cdots \wedge g_{i_{m-n}}$ be two vectors in $\wedge^{m-n} \mathbb{E}_{t}^{m}$, where $\left\{f_{1}, f_{2}, \ldots, f_{m}\right\}$ and $\left\{g_{1}, g_{2}, \ldots, g_{m}\right\}$ are two orthonormal bases of $\mathbb{E}_{t}^{m}$. Define an indefinite inner product $\langle\langle\rangle$,$\rangle on \bigwedge^{m-n} \mathbb{E}_{t}^{m}$ by

$$
\left\langle\left\langle f_{i_{1}} \wedge \cdots \wedge f_{i_{m-n}}, g_{i_{1}} \wedge \cdots \wedge g_{i_{m-n}}\right\rangle\right\rangle=\operatorname{det}\left(\left\langle f_{i_{\ell}}, g_{j_{k}}\right\rangle\right) .
$$

Therefore, for some positive integer $s$, we may identify $\bigwedge^{m-n} \mathbb{E}_{t}^{m}$ with some pseudo-Euclidean space $\mathbb{E}_{s}^{N}$, where $N=\left(\begin{array}{c}m \\ m-n\end{array}\right)$. The map $\nu: M \rightarrow G(m-$ 
$n, m) \subset \mathbb{E}_{s}^{N}$ from an oriented pseudo-Riemannian submanifold $M$ into $G(m-$ $n, m)$ defined by

$$
\nu(p)=\left(e_{n+1} \wedge e_{n+2} \wedge \cdots \wedge e_{m}\right)(p)
$$

is called the Gauss map of $M$ which assigns to a point $p$ in $M$ the oriented $(m-n)$-plane through the origin of $\mathbb{E}_{t}^{m}$ and parallel to the normal space of $M$ at $p,[17]$.

We put $\varepsilon=\langle\langle\nu, \nu\rangle\rangle=\varepsilon_{n+1} \varepsilon_{n+2} \cdots \varepsilon_{m}= \pm 1$ and

$$
\widetilde{M}_{s}^{N-1}(\varepsilon)=\left\{\begin{array}{lll}
\mathbb{S}_{s}^{N-1}(1) & \text { in } \mathbb{E}_{s}^{N} & \text { if } \varepsilon=1 \\
\mathbb{H}_{s-1}^{N-1}(-1) & \text { in } \mathbb{E}_{s}^{N} & \text { if } \varepsilon=-1
\end{array}\right.
$$

Then the Gauss image $\nu(M)$ can be viewed as $\nu(M) \subset \widetilde{M}_{s}^{N-1}(\varepsilon)$.

Lemma 2.1. Let $M$ be an n-dimensional submanifold of a pseudo-Euclidean space $\mathbb{E}_{t}^{n+2}$. Then, the Laplacian of the Gauss map $\nu=e_{n+1} \wedge e_{n+2}$ is given by

$$
\begin{aligned}
\Delta \nu= & \|h\|^{2} \nu+2 \sum_{j<k} \varepsilon_{j} \varepsilon_{k} R^{D}\left(e_{j}, e_{k} ; e_{n+1}, e_{n+2}\right) e_{j} \wedge e_{k} \\
& +\nabla\left(\operatorname{tr} A_{n+1}\right) \wedge e_{n+2}+e_{n+1} \wedge \nabla\left(\operatorname{tr} A_{n+2}\right) \\
& +n \sum_{j=1}^{n} \varepsilon_{j} \omega_{(n+1)(n+2)}\left(e_{j}\right) H \wedge e_{j},
\end{aligned}
$$

where $\|h\|^{2}$ is the squared length of the second fundamental form, $R^{D}$ the normal curvature tensor, and $\nabla\left(\operatorname{tr} A_{r}\right)$ the gradient of $\operatorname{tr} A_{r}$.

Let $M$ be a surface in the pseudo-Euclidean space $\mathbb{E}_{2}^{4}$. We choose a local orthonormal frame field $\left\{e_{1}, e_{2}, e_{3}, e_{4}\right\}$ on $M$ such that $e_{1}, e_{2}$ are tangent to $M$, and $e_{3}, e_{4}$ are normal to $M$. Let $C$ be a vector field in $\bigwedge^{2} \mathbb{E}_{2}^{4} \equiv \mathbb{E}_{4}^{6}$. Since the set $\left\{e_{A} \wedge e_{B} \mid 1 \leq A<B \leq 4\right\}$ is an orthonormal basis for $\mathbb{E}_{4}^{6}$, the vector $C$ can be expressed as

$$
C=\sum_{1 \leq A<B \leq 4} \varepsilon_{A} \varepsilon_{B} C_{A B} e_{A} \wedge e_{B},
$$

where $C_{A B}=\left\langle\left\langle C, e_{A} \wedge e_{B}\right\rangle\right\rangle$.

Lemma 2.2. A vector $C$ in $\Lambda^{2} \mathbb{E}_{2}^{4} \equiv \mathbb{E}_{4}^{6}$ written by (2.8) is constant if and only if the following equations are satisfied for $i=1,2$

$$
\begin{aligned}
& e_{i}\left(C_{12}\right)=\varepsilon_{3} h_{i 2}^{3} C_{13}+\varepsilon_{4} h_{i 2}^{4} C_{14}-\varepsilon_{3} h_{i 1}^{3} C_{23}-\varepsilon_{4} h_{i 1}^{4} C_{24}, \\
& e_{i}\left(C_{13}\right)=-\varepsilon_{2} h_{i 2}^{3} C_{12}+\varepsilon_{4} \omega_{34}\left(e_{i}\right) C_{14}+\varepsilon_{2} \omega_{12}\left(e_{i}\right) C_{23}-\varepsilon_{4} h_{i 1}^{4} C_{34}, \\
& e_{i}\left(C_{14}\right)=-\varepsilon_{2} h_{i 2}^{4} C_{12}-\varepsilon_{3} \omega_{34}\left(e_{i}\right) C_{13}+\varepsilon_{2} \omega_{12}\left(e_{i}\right) C_{24}+\varepsilon_{3} h_{i 1}^{3} C_{34}, \\
& e_{i}\left(C_{23}\right)=\varepsilon_{1} h_{i 1}^{3} C_{12}-\varepsilon_{1} \omega_{12}\left(e_{i}\right) C_{13}+\varepsilon_{4} \omega_{34}\left(e_{i}\right) C_{24}-\varepsilon_{4} h_{i 2}^{4} C_{34}, \\
& e_{i}\left(C_{24}\right)=\varepsilon_{1} h_{i 1}^{4} C_{12}-\varepsilon_{1} \omega_{12}\left(e_{i}\right) C_{14}-\varepsilon_{3} \omega_{34}\left(e_{i}\right) C_{23}+\varepsilon_{3} h_{i 2}^{3} C_{34}, \\
& e_{i}\left(C_{34}\right)=\varepsilon_{1} h_{i 1}^{4} C_{13}-\varepsilon_{1} h_{i 1}^{3} C_{14}+\varepsilon_{2} h_{i 2}^{4} C_{23}-\varepsilon_{2} h_{i 2}^{3} C_{24} .
\end{aligned}
$$




\section{$3 \quad$ Rotational Surfaces IN $\mathbb{E}_{2}^{4}$}

In this section, we focus on rotational surfaces in $\mathbb{E}_{2}^{4}$ with profile curves which lie in 2-dimensional planes, and we obtain some geometric quantities about these surfaces.

Let $M_{1}(b)$ and $M_{2}(b)$ be rotational surfaces in the pseudo-Euclidean space $\mathbb{E}_{2}^{4}$ whose profile curves lie in 2 -planes. These rotational surfaces defined below are invariant under some rotation subgroup of rotation group in $\mathbb{E}_{2}^{4}$. We can choose a profile curve $\alpha$ of $M_{1}(b)$ in the $y w$-plane as $\alpha(u)=(0, y(u), 0, w(u))$, defined on an open subset $I$ of $\mathbb{R}$ and thus the parametrization of $M_{1}(b)$ is given by

$$
M_{1}(b): r_{1}(u, v)=(w(u) \sinh v, y(u) \cosh (b v), y(u) \sinh (b v), w(u) \cosh v)
$$

with some constant $b>0$, where $u \in I$ is an open subset of $\mathbb{R}$ and $v \in \mathbb{R}$.

We consider the following orthonormal moving frame field $e_{1}, e_{2}, e_{3}, e_{4}$ on $M_{1}(b)$ in $\mathbb{E}_{2}^{4}$ such that $e_{1}, e_{2}$ are tangent to $M_{1}(b)$, and $e_{3}, e_{4}$ are normal to $M_{1}(b)$ :

$$
\begin{aligned}
& e_{1}=\frac{1}{q} \frac{\partial}{\partial v}, \quad e_{2}=\frac{1}{A} \frac{\partial}{\partial u}, \\
& e_{3}=\frac{1}{A}\left(y^{\prime}(u) \sinh v, w^{\prime}(u) \cosh (b v), w^{\prime}(u) \sinh (b v), y^{\prime}(u) \cosh v\right), \\
& e_{4}=-\frac{\varepsilon \varepsilon^{*}}{q}(b y(u) \cosh v, w(u) \sinh (b v), w(u) \cosh (b v), b y(u) \sinh v),
\end{aligned}
$$

where $A=\sqrt{\varepsilon\left(y^{\prime 2}(u)-w^{\prime 2}(u)\right)} \neq 0, q=\sqrt{\varepsilon^{*}\left(w^{2}(u)-b^{2} y^{2}(u)\right)} \neq 0$, and $\varepsilon=\operatorname{sgn}\left(y^{\prime 2}(u)-w^{\prime 2}(u)\right), \varepsilon^{*}=\operatorname{sgn}\left(w^{2}(u)-b^{2} y^{2}(u)\right)$. Then $\varepsilon_{1}=-\varepsilon_{4}=\varepsilon^{*}, \varepsilon_{2}=$ $-\varepsilon_{3}=\varepsilon$.

By a direct calculation, we have the components of the second fundamental form and the connection forms as

$$
\begin{aligned}
h_{11}^{3} & =\frac{1}{A q^{2}}\left(b^{2} y(u) w^{\prime}(u)-w(u) y^{\prime}(u)\right), h_{22}^{3}=\frac{1}{A^{3}}\left(w^{\prime}(u) y^{\prime \prime}(u)-y^{\prime}(u) w^{\prime \prime}(u)\right) \\
h_{12}^{4} & =\frac{\varepsilon \varepsilon^{*} b}{A q^{2}}\left(w(u) y^{\prime}(u)-y(u) w^{\prime}(u)\right), \quad h_{12}^{3}=h_{11}^{4}=h_{22}^{4}=0 \\
\omega_{12}\left(e_{1}\right) & =\frac{1}{A q^{2}}\left(b^{2} y(u) y^{\prime}(u)-w(u) w^{\prime}(u)\right), \quad \omega_{12}\left(e_{2}\right)=0 \\
\omega_{34}\left(e_{1}\right) & =\frac{\varepsilon \varepsilon^{*} b}{A q^{2}}\left(w(u) w^{\prime}(u)-y(u) y^{\prime}(u)\right), \quad \omega_{34}\left(e_{2}\right)=0
\end{aligned}
$$

For the rotational surface $M_{2}(b)$, we can choose a profile curve $\beta$ in the $x z-$ plane as $\beta(u)=(x(u), 0, z(u), 0)$ defined on an open subset $I$ of $\mathbb{R}$, and thus the parametrization of $M_{2}(b)$ is given by

$$
M_{2}(b): r_{2}(u, v)=(x(u) \cos v, x(u) \sin v, z(u) \cos (b v), z(u) \sin (b v))
$$


with some constant $b>0$, where $u \in I$ is an open subset of $\mathbb{R}$ and $v \in(0,2 \pi)$.

We consider the following orthonormal moving frame fields $e_{1}, e_{2}, e_{3}, e_{4}$ on $M_{2}(b)$ in $\mathbb{E}_{2}^{4}$ such that $e_{1}, e_{2}$ are tangent to $M_{2}(b)$, and $e_{3}, e_{4}$ are normal to $M_{2}(b)$ :

$$
\begin{aligned}
& e_{1}=\frac{1}{\bar{q}} \frac{\partial}{\partial v}, \quad e_{2}=\frac{1}{\bar{A}} \frac{\partial}{\partial u}, \\
& e_{3}=\frac{1}{\bar{A}}\left(z^{\prime}(u) \cos v, z^{\prime}(u) \sin v, x^{\prime}(u) \cos (b v), x^{\prime}(u) \sin (b v)\right), \\
& e_{4}=-\frac{\varepsilon \varepsilon^{*}}{\bar{q}}(b z(u) \sin v,-b z(u) \cos v, x(u) \sin (b v),-x(u) \cos (b v)),
\end{aligned}
$$

where $\bar{A}=\sqrt{\varepsilon\left(x^{\prime 2}(u)-{z^{\prime}}^{2}(u)\right)} \neq 0, \bar{q}=\sqrt{\varepsilon^{*}\left(x^{2}(u)-b^{2} z^{2}(u)\right)} \neq 0$, and $\varepsilon=$ $\operatorname{sgn}\left(x^{\prime 2}(u)-z^{\prime 2}(u)\right), \varepsilon^{*}=\operatorname{sgn}\left(x^{2}(u)-b^{2} z^{2}(u)\right)$. Then $\varepsilon_{1}=-\varepsilon_{4}=\varepsilon^{*}, \varepsilon_{2}=$ $-\varepsilon_{3}=\varepsilon$.

By a direct computation, we have the components of the second fundamental form and the connection forms as

$$
\begin{aligned}
h_{11}^{3} & =\frac{1}{\bar{A} \bar{q}^{2}}\left(b^{2} z(u) x^{\prime}(u)-x(u) z^{\prime}(u)\right), h_{22}^{3}=\frac{1}{\bar{A}^{3}}\left(z^{\prime}(u) x^{\prime \prime}(u)-x^{\prime}(u) z^{\prime \prime}(u)\right), \\
h_{12}^{4} & =\frac{\varepsilon \varepsilon^{*} b}{\bar{A} \bar{q}^{2}}\left(z(u) x^{\prime}(u)-x(u) z^{\prime}(u)\right), \quad h_{12}^{3}=h_{11}^{4}=h_{22}^{4}=0, \\
\omega_{12}\left(e_{1}\right) & =\frac{1}{\bar{A} \bar{q}^{2}}\left(b^{2} z(u) z^{\prime}(u)-x(u) x^{\prime}(u)\right), \quad \omega_{12}\left(e_{2}\right)=0, \\
\omega_{34}\left(e_{1}\right) & =\frac{\varepsilon \varepsilon^{*} b}{\bar{A} \bar{q}^{2}}\left(z(u) z^{\prime}(u)-x(u) x^{\prime}(u)\right), \quad \omega_{34}\left(e_{2}\right)=0 .
\end{aligned}
$$

Therefore, we have the mean curvature vector $H$, Gaussian curvature $K$ and normal curvature $R^{D}$ for the rotational surfaces for $M_{1}(b)$ and $M_{2}(b)$ as follows

$$
\begin{aligned}
H & =-\frac{1}{2}\left(\varepsilon \varepsilon^{*} h_{11}^{3}+h_{22}^{3}\right) e_{3}, \\
K & =\varepsilon^{*}\left(h_{12}^{4}\right)^{2}-\varepsilon h_{11}^{3} h_{22}^{3}, \\
R^{D}\left(e_{1}, e_{2} ; e_{3}, e_{4}\right) & =h_{12}^{4}\left(\varepsilon h_{22}^{3}-\varepsilon^{*} h_{11}^{3}\right) .
\end{aligned}
$$

On the other hand, by using the Codazzi equation (2.3) we obtain

$$
\begin{aligned}
& e_{2}\left(h_{11}^{3}\right)=\varepsilon^{*} h_{12}^{4} \omega_{34}\left(e_{1}\right)+\omega_{12}\left(e_{1}\right)\left(\varepsilon^{*} h_{11}^{3}-\varepsilon h_{22}^{3}\right), \\
& e_{2}\left(h_{12}^{4}\right)=-\varepsilon h_{22}^{3} \omega_{34}\left(e_{1}\right)+2 \varepsilon^{*} h_{12}^{4} \omega_{12}\left(e_{1}\right) .
\end{aligned}
$$

The rotational surfaces $M_{1}(b)$ and $M_{2}(b)$ defined by (3.1) and (3.9) for $b=1$, $x(u)=y(u)=f(u) \sinh u$ and $z(u)=w(u)=f(u) \cosh u$ are also known as Vranceanu rotational surface, where $f(u)$ is a smooth function, [15, 17]. 


\section{Pseudo-Umbilical Rotational SuRfaCES in $\mathbb{E}_{2}^{4}$}

In this section, we obtain all pseudo-umbilical rotational surfaces $M_{1}(b)$ and $M_{2}(b)$ in $\mathbb{E}_{2}^{4}$ defined by (3.1) and (3.9).

By the definition of pseudo-umbilical surface and (3.17), the rotational surfaces $M_{1}(b)$ and $M_{2}(b)$ are pseudo-umbilical if and only if $\varepsilon^{*} h_{11}^{3}=\varepsilon h_{22}^{3}$.

Hence, from (3.5) the surface $M_{1}(b)$ is pseudo-umbilical if and only if the component functions $y(u)$ and $w(u)$ of the profile curve $\alpha$ satisfy the following differential equation

$$
w^{\prime}(u) y^{\prime \prime}(u)-y^{\prime}(u) w^{\prime \prime}(u)-\left(y^{\prime 2}(u)-w^{\prime 2}(u)\right) \frac{b^{2} y(u) w^{\prime}(u)-w(u) y^{\prime}(u)}{w^{2}(u)-b^{2} y^{2}(u)}=0 .
$$

By a simple computation, it can be shown that a non-planar rotational surface $M_{1}(b)$ in $\mathbb{E}_{2}^{4}$ defined by (3.1) for $b=1$ is pseudo-umbilical if and only if its profile curve is given by

$$
w(u)+y(u)=\lambda_{0}(w(u)-y(u))^{\mu_{0}}
$$

for some constants $\lambda_{0} \neq 0$ and $\mu_{0}$ such that $(w(u)-y(u))^{\mu_{0}}$ is real valued.

If $\mu_{0}=1$ and $\lambda_{0}^{2} \neq 1$, from (4.2) we have $y(u)=\frac{\lambda_{0}-1}{\lambda_{0}+1} w(u)$, that is, the profile curve $\alpha$ is a part of line passing through the origin. It can be shown easily that $M_{1}(1)$ is an open part of a timelike plane in $\mathbb{E}_{2}^{4}$.

If $\mu_{0}=-1$, then (4.2) implies that $w^{2}(u)-y^{2}(u)=\lambda_{0}$ which gives $(\mathrm{a}-5)$ and $(\mathrm{a}-6)$ in Theorem 4.2 for $b=1$.

From equations (3.5), (3.6) we obtain $h_{12}^{4}=-\varepsilon \varepsilon^{*} h_{11}^{3}$ in the case $b=1$. Also, we know that such a relation $\varepsilon^{*} h_{11}^{3}=\varepsilon h_{22}^{3}$ exists. Hence, from the equation (3.18) we conclude:

Proposition 4.1. Let $M_{1}(1)$ be a rotational surface in $\mathbb{E}_{2}^{4}$ given by (3.1). Then, $M_{1}(1)$ is pseudo-umbilical if and only if $M_{1}(1)$ is flat.

In [17], flat Vranceanu surfaces which are pseudo-umbilical surfaces $M_{1}(1)$ were studied for different purposes. It was proven that the Vranceanu rotational surface is flat if $f(u)=\lambda e^{\mu u}$, where $\lambda$ and $\mu$ are real numbers. Then, we have $\varepsilon^{*}=\operatorname{sgn}\left(\lambda^{2} e^{2 \mu u}\right)=1$ and $\varepsilon=\operatorname{sgn}\left(\lambda^{2}\left(1-\mu^{2}\right) e^{2 \mu u}\right)=1$ for $|\mu|<1$ and $\varepsilon=-1$ for $|\mu|>1$. Thus, the Vranceanu rotational surface is spacelike pseudo-umbilical for $|\mu|<1$ and timelike pseudo-umbilical for $|\mu|>1$.

For $c_{0} \neq 0$ and $\theta>0$, we define the following functions

$$
\Phi\left(\theta, b, \varepsilon, \varepsilon^{*}\right)=\int_{0}^{\theta} \sqrt{\frac{\varepsilon^{*} c_{0}^{2}\left(\sinh ^{2} \eta-b^{2} \cosh ^{2} \eta\right)}{\varepsilon^{*} c_{0}^{2}\left(\sinh ^{2} \eta-b^{2} \cosh ^{2} \eta\right)-\varepsilon}} d \eta
$$

and

$$
\Omega\left(\theta, b, \varepsilon, \varepsilon^{*}\right)=\int_{0}^{\theta} \sqrt{\frac{\varepsilon^{*} c_{0}^{2}\left(\cosh ^{2} \eta-b^{2} \sinh ^{2} \eta\right)}{\varepsilon^{*} c_{0}^{2}\left(\cosh ^{2} \eta-b^{2} \sinh ^{2} \eta\right)+\varepsilon}} d \eta .
$$

such that the integrands are real valued functions. 
Theorem 4.2. Let $M_{1}(b)$ be a non-planar rotational surface in the pseudoEuclidean space $\mathbb{E}_{2}^{4}$ given by (3.1). Then,

(a) $M_{1}(b)$ is a spacelike pseudo-umbilical surface in $\mathbb{E}_{2}^{4}$ if and only if the component functions of the unit speed profile curve $\alpha$ of $M_{1}(b)$ are given by one of the followings:

$(a-1)$

$$
y(\theta)=c e^{\psi(\theta)} \cosh \theta \text { and } w(\theta)=c e^{\psi(\theta)} \sinh \theta,
$$

where $\psi(\theta)=\Phi(\theta, b, 1,1), 0<b<1$ and $c_{0}^{2}\left(\sinh ^{2} \theta-b^{2} \cosh ^{2} \theta\right)>1$ for some $c_{0} \in \mathbb{R}$ and $c \in \mathbb{R}_{+}$;

$$
y(\theta)=c e^{\psi(\theta)} \cosh \theta \text { and } w(\theta)=c e^{\psi(\theta)} \sinh \theta,
$$

where $\psi(\theta)=\Phi(\theta, b,-1,-1), b \geq 1$ and $c \in \mathbb{R}_{+}$. In this case, the surface $M_{1}(b)$ has negative definite metric;

$$
y(\theta)=c e^{\varphi(\theta)} \sinh \theta \text { and } w(\theta)=c e^{\varphi(\theta)} \cosh \theta,
$$

where $\varphi(\theta)=\Omega(\theta, b, 1,1), 0<b \leq 1$ and $c \in \mathbb{R}_{+}$;

$(a-4)$

$$
y(\theta)=c e^{\varphi(\theta)} \sinh \theta \text { and } w(\theta)=c e^{\varphi(\theta)} \cosh \theta,
$$

where $\varphi(\theta)=\Omega(\theta, b,-1,-1), b>1$ and $c_{0}^{2}\left(b^{2} \sinh ^{2} \theta-\cosh ^{2} \theta\right)>1$ for some $c_{0} \in \mathbb{R}$ and $c \in \mathbb{R}_{+}$. In this case, the surface $M_{1}(b)$ has negative definite metric;

$$
y(\theta)=r_{0} \sinh \theta \text { and } w(\theta)=r_{0} \cosh \theta,
$$

where $r_{0}$ is non-zero constant and $0<b \leq 1$. In this case, the surface $M_{1}(b)$ lies in $\mathbb{H}_{1}^{3}\left(-r_{0}^{-2}\right) \subset \mathbb{E}_{2}^{4}$;

$(a-6)$

$$
y(\theta)=r_{0} \cosh \theta \text { and } w(\theta)=r_{0} \sinh \theta,
$$

where $r_{0}$ is non-zero constant and $b \geq 1$. In this case, the surface $M_{1}(b)$ has negative definite metric and is lying in $\mathbb{S}_{2}^{3}\left(r_{0}^{-2}\right) \subset \mathbb{E}_{2}^{4}$.

(b) $M_{1}(b)$ is a timelike pseudo-umbilical surface in $\mathbb{E}_{2}^{4}$ if and only if the component functions of the unit speed profile curve $\alpha$ of $M_{1}(b)$ are given by one of the followings:

$$
y(\theta)=c e^{\psi(\theta)} \cosh \theta \text { and } w(\theta)=c e^{\psi(\theta)} \sinh \theta,
$$

where $\psi(\theta)=\Phi(\theta, b, 1,-1), b \geq 1$ and $c_{0}^{2}\left(b^{2} \cosh ^{2} \theta-\sinh ^{2} \theta\right)>1$ for some $c_{0} \in \mathbb{R}$ and $c \in \mathbb{R}_{+}$;

$$
y(\theta)=c e^{\psi(\theta)} \cosh \theta \text { and } w(\theta)=c e^{\psi(\theta)} \sinh \theta,
$$


where $\psi(\theta)=\Phi(\theta, b,-1,1), 0<b<1$ and $c \in \mathbb{R}_{+}$;

$$
y(\theta)=c e^{\varphi(\theta)} \sinh \theta \text { and } w(\theta)=c e^{\varphi(\theta)} \cosh \theta,
$$

where $\varphi(\theta)=\Omega(\theta, b, 1,-1), b>1$ and $c \in \mathbb{R}_{+}$;

$(b-4)$

$$
y(\theta)=c e^{\varphi(\theta)} \sinh \theta \text { and } w(\theta)=c e^{\varphi(\theta)} \cosh \theta,
$$

where $\varphi(\theta)=\Omega(\theta, b,-1,1), 0<b \leq 1$ and $c_{0}^{2}\left(\cosh ^{2} \theta-b^{2} \sinh ^{2} \theta\right)>1$ for some $c_{0} \in \mathbb{R}$ and $c \in \mathbb{R}_{+}$;

$$
y(\theta)=r_{0} \sinh \theta \text { and } w(\theta)=r_{0} \cosh \theta,
$$

where $r_{0}$ is non-zero constant and $b>1$. In this case, the surface $M_{1}(b)$ lies in $\mathbb{H}_{1}^{3}\left(-r_{0}^{-2}\right) \subset \mathbb{E}_{2}^{4}$;

$$
y(\theta)=r_{0} \cosh \theta \text { and } w(\theta)=r_{0} \sinh \theta,
$$

where $r_{0}$ is non-zero constant and $0<b<1$. In this case, the surface $M_{1}(b)$ lies in $\mathbb{S}_{2}^{3}\left(r_{0}^{-2}\right) \subset \mathbb{E}_{2}^{4}$.

Proof. Let $M_{1}(b)$ be a rotational surface in the pseudo-Euclidean space $\mathbb{E}_{2}^{4}$ given by (3.1). From (3.7) and (3.8), it is seen that $\omega_{12}\left(e_{1}\right)$ and $\omega_{34}\left(e_{1}\right)$ are functions of $u$, and $\omega_{12}\left(e_{2}\right)=\omega_{34}\left(e_{2}\right)=0$. By using these facts and (3.19), we have

$$
-e_{2}\left(\omega_{34}\left(e_{1}\right)\right)+\varepsilon^{*} \omega_{12}\left(e_{1}\right) \omega_{34}\left(e_{1}\right)=h_{12}^{4}\left(\varepsilon h_{22}^{3}-\varepsilon^{*} h_{11}^{3}\right) .
$$

Now, assume that $M_{1}(b)$ is pseudo-umbilical surface, i.e., $\varepsilon^{*} h_{11}^{3}=\varepsilon h_{22}^{3}$. Then (4.5) implies

$$
e_{2}\left(\omega_{34}\left(e_{1}\right)\right)-\varepsilon^{*} \omega_{12}\left(e_{1}\right) \omega_{34}\left(e_{1}\right)=0 .
$$

This equation together with the second equation in (3.2) and the first equation in (3.7) gives

$$
\frac{d}{d u}\left(\omega_{34}\left(e_{1}\right)\right)=-\frac{w(u) w^{\prime}(u)-b^{2} y(u) y^{\prime}(u)}{w^{2}(u)-b^{2} y^{2}(u)} \omega_{34}\left(e_{1}\right) .
$$

It is clear that $\omega_{34}\left(e_{1}\right)=0$ is a solution of (4.7). In this case, from (3.8) we have $w(u) w^{\prime}(u)-y(u) y^{\prime}(u)=0$ which implies that $w^{2}(u)-y^{2}(u)=\lambda_{0}$, for non-zero constant $\lambda_{0}$.

For $\lambda_{0}=r_{0}^{2}>0$, we put $y(u)=r_{0} \sinh \theta(u)$ and $w(u)=r_{0} \cosh \theta(u)$, where $\theta(u)$ is a smooth function with $\theta^{\prime}(u) \neq 0$. So, $\varepsilon=\operatorname{sgn}\left(r_{0}^{2} \theta^{\prime 2}(u)\right)=1$ and $\varepsilon^{*}=\operatorname{sgn}\left(r_{0}^{2}\left(\cosh ^{2} \theta(u)-b^{2} \sinh ^{2} \theta(u)\right)\right)=1$ for $0<b \leq 1$ and $\varepsilon^{*}=-1$ for $b>1$. Therefore, for $0<b \leq 1, M_{1}(b)$ is a spacelike pseudo-umbilical surface which gives (a-5), and for $b>1, M_{1}(b)$ is a timelike pseudo-umbilical surface which gives (b-5). Moreover, $M_{1}(b)$ lies in $\mathbb{H}_{1}^{3}\left(-r_{0}{ }^{-2}\right) \subset \mathbb{E}_{2}^{4}$.

For $\lambda_{0}=-r_{0}^{2}<0$, we put $y(u)=r_{0} \cosh \theta(u)$ and $w(u)=r_{0} \sinh \theta(u)$, where $\theta(u)$ is a smooth function with $\theta^{\prime}(u) \neq 0$. So $\varepsilon=\operatorname{sgn}\left(-r_{0}^{2} \theta^{2}(u)\right)=-1$, 
and $\varepsilon^{*}=\operatorname{sgn}\left(r_{0}^{2}\left(\sinh ^{2} \theta(u)-b^{2} \cosh ^{2} \theta(u)\right)\right)=-1$ for $b \geq 1$ and $\varepsilon^{*}=1$ for $0<b<1$. Then, for $b \geq 1, M_{1}(b)$ is a spacelike pseudo-umbilical surface with negative definite metric which gives $(\mathrm{a}-6)$, and for $0<b<1, M_{1}(b)$ is a timelike pseudo-umbilical surface which gives (b-6). Moreover, $M_{1}(b)$ lies in $\mathbb{S}_{2}^{3}\left(r_{0}{ }^{-2}\right) \subset \mathbb{E}_{2}^{4}$.

Let $\omega_{34} \neq 0$ on $M_{1}$. By combining (3.8) and (4.7) we have

$$
\frac{\varepsilon \varepsilon^{*} b\left(w(u) w^{\prime}(u)-y(u) y^{\prime}(u)\right)}{\sqrt{\varepsilon^{*}\left(w^{2}(u)-b^{2} y^{2}(u)\right)} \sqrt{\varepsilon\left(y^{\prime 2}(u)-w^{\prime 2}(u)\right)}}=b_{0}
$$

for some constant $b_{0} \neq 0$.

Now we suppose that the profile curve $\alpha$ is a unit speed curve, that is, $y^{\prime 2}(u)-w^{\prime 2}(u)=\varepsilon$. Thus equation (4.8) becomes

$$
\frac{w(u) w^{\prime}(u)-y(u) y^{\prime}(u)}{\sqrt{\varepsilon^{*}\left(w^{2}(u)-b^{2} y^{2}(u)\right)}}=c_{0}
$$

for some constant $c_{0} \neq 0$.

Without loss of generality, firstly we choose $y(u)=r(u) \cosh \theta(u)$ and $w(u)=$ $r(u) \sinh \theta(u)$. Then, from ${y^{\prime}}^{2}(u)-{w^{\prime}}^{2}(u)=\varepsilon$ and (4.9) we have, respectively,

$$
\varepsilon d u^{2}=d r^{2}-r^{2} d \theta^{2} \text { and } d u=-\frac{d r}{c_{0} \sqrt{\varepsilon^{*}\left(\sinh ^{2} \theta-b^{2} \cosh ^{2} \theta\right)}}
$$

from which we obtain that

$$
\frac{d r}{r}=\sqrt{\frac{\varepsilon^{*} c_{0}^{2}\left(\sinh ^{2} \theta-b^{2} \cosh ^{2} \theta\right)}{\varepsilon^{*} c_{0}^{2}\left(\sinh ^{2} \theta-b^{2} \cosh ^{2} \theta\right)-\varepsilon}} d \theta,
$$

where $\varepsilon^{*} c_{0}^{2}\left(\sinh ^{2} \theta-b^{2} \cosh ^{2} \theta\right)>\varepsilon$. The integration of (4.10) gives

$$
r(\theta)=c e^{\Phi\left(\theta, b, \varepsilon, \varepsilon^{*}\right)},
$$

where $\Phi\left(\theta, b, \varepsilon, \varepsilon^{*}\right)$ is defined by (4.3) and $c \in \mathbb{R}_{+}$. From $\varepsilon^{*}=\operatorname{sgn}\left(r^{2}(u)\left(\sinh ^{2} \theta(u)-\right.\right.$ $\left.b^{2} \cosh ^{2} \theta(u)\right)$ ), we get $\varepsilon^{*}=1$ for $0<b<1$, and $\varepsilon^{*}=-1$ for $b \geq 1$. Now, by (4.11) and (4.3) we have (a-1) if $\varepsilon=\varepsilon^{*}=1$, and the integrand in (4.11) is defined for $c_{0}^{2}\left(\sinh ^{2} \theta-b^{2} \cosh ^{2} \theta\right)>1$ for some $c_{0} \in \mathbb{R}$; (a-2) if $\varepsilon=\varepsilon^{*}=-1$; (b-1) if $\varepsilon=-\varepsilon^{*}=1$, and the integrand in (4.3) is defined for $c_{0}^{2}\left(b^{2} \cosh ^{2} \theta-\sinh ^{2} \theta\right)>1$ for some $c_{0} \in \mathbb{R} ;(\mathrm{b}-2)$ if $\varepsilon^{*}=-\varepsilon=1$..

Secondly, let $y(u)=r(u) \sinh \theta(u)$ and $w(u)=r(u) \cosh \theta(u)$. By a similar calculation we obtain that

$$
r(\theta)=c e^{\Omega\left(\theta, b, \varepsilon, \varepsilon^{*}\right)},
$$

where $\Omega\left(\theta, b, \varepsilon, \varepsilon^{*}\right)$ is defined by (4.4) and $c \in \mathbb{R}_{+}$. From $\varepsilon^{*}=\operatorname{sgn}\left(r^{2}(u)\left(\cosh ^{2} \theta(u)-\right.\right.$ $\left.b^{2} \sinh ^{2} \theta(u)\right)$ ) we get $\varepsilon^{*}=1$ for $0<b \leq 1$, and $\varepsilon^{*}=-1$ for $b>1$. Now by considering (4.12) and (4.4) we have (a-3) if $\varepsilon=\varepsilon^{*}=1$, and (a-4) if $\varepsilon=\varepsilon^{*}=-1$ 
and the integrand in (4.4) is defined for $c_{0}^{2}\left(b^{2} \sinh ^{2} \theta-\cosh ^{2} \theta\right)>1$ for some $c_{0} \in \mathbb{R} ;(\mathrm{b}-3)$ if $\varepsilon=-\varepsilon^{*}=1$, and $(\mathrm{b}-4)$ if $\varepsilon^{*}=-\varepsilon=1$ and the integrand in (4.4) is defined for $c_{0}^{2}\left(\cosh ^{2} \theta-b^{2} \sinh ^{2} \theta\right)>1$ for some $c_{0} \in \mathbb{R}$.

Conversely, we assume that $y(\theta)$ and $w(\theta)$ are given by $y(\theta)=c e^{\Phi\left(\theta, b, \varepsilon, \varepsilon^{*}\right)} \cosh \theta$ and $w(\theta)=c e^{\Phi\left(\theta, b, \varepsilon, \varepsilon^{*}\right)} \sinh \theta$ for the function $\Phi$ defined by (4.3). Since $y(\theta)$ and $w(\theta)$ satisfy (4.6), equation (4.5) implies that either $h_{12}^{4}=0$ or $\varepsilon^{*} h_{11}^{3}=\varepsilon h_{22}^{3}$. From the first equation in (3.6) we have $h_{12}^{4} \neq 0$ as $\frac{d \theta}{d u} \neq 0$, and thus $\varepsilon^{*} h_{11}^{3}=$ $\varepsilon h_{22}^{3}$. In the case, the profile curve $\alpha$ given by $y(\theta)=c e^{\Omega\left(\theta, b, \varepsilon, \varepsilon^{*}\right)} \sinh \theta$ and $w(\theta)=c e^{\Omega\left(\theta, b, \varepsilon, \varepsilon^{*}\right)} \cosh \theta$ for the function $\Omega$ defined by (4.4), by a similar argument it can be seen that $\varepsilon^{*} h_{11}^{3}=\varepsilon h_{22}^{3}$. Therefore $M_{1}(b)$ is a pseudo-umbilical surface in the pseudo-Euclidean space $\mathbb{E}_{2}^{4}$.

Similarly, we determine pseudo-umbilical rotational surface $M_{2}(b)$ in $\mathbb{E}_{2}^{4}$ given by (3.9). From (3.13), the surface $M_{2}(b)$ is pseudo-umbilical if and only if the component functions $x(u)$ and $z(u)$ of the profile curve $\beta$ satisfy the differential equation

$$
z^{\prime}(u) x^{\prime \prime}(u)-x^{\prime}(u) z^{\prime \prime}(u)-\left(x^{\prime 2}(u)-z^{\prime 2}(u)\right) \frac{b^{2} z(u) x^{\prime}(u)-x(u) z^{\prime}(u)}{x^{2}(u)-b^{2} z^{2}(u)}=0 .
$$

By a simple computation, it can be shown that a non-planar rotational surface $M_{2}(b)$ in $\mathbb{E}_{2}^{4}$ defined by $(3.9)$ for $b=1$ is pseudo-umbilical if and only if its profile curve is given by

$$
z(u)-x(u)=\lambda_{0}(z(u)+x(u))^{\mu_{0}}
$$

for some constants $\lambda_{0} \neq 0$ and $\mu_{0}$ such that $(z(u)+x(u))^{\mu_{0}}$ is real valued.

If $\mu_{0}=1$ and $\lambda_{0}^{2} \neq 1$, from (4.14) we have $x(u)=\frac{1-\lambda_{0}}{1+\lambda_{0}} z(u)$, that is the profile curve $\beta$ is a part of a line passing through the origin. It can be shown easily that $M_{2}(1)$ is an open part of a spacelike plane in $\mathbb{E}_{2}^{4}$.

If $\mu_{0}=-1$, then (4.14) implies that $z^{2}(u)-x^{2}(u)=\lambda_{0}$ which gives $(\mathrm{b}-5)$ and (b-6) in Theorem 4.4 for $b=1$.

Because of the similar reason for the rotational surface $M_{1}(b)$, we have the following:

Proposition 4.3. Let $M_{2}(1)$ be a rotational surface in $\mathbb{E}_{2}^{4}$ given by (3.9). Then, $M_{2}(1)$ is pseudo-umbilical if and only if $M_{2}(1)$ is flat.

In [17], it was shown that the Vranceanu rotational surface is flat if $f(u)=$ $\lambda e^{\mu u}$, where $\lambda$ and $\mu$ are real numbers. For the function $f(u)$, the component function $x(u)$ and $z(u)$ satisfy the solution (4.14). Moreover, $\varepsilon^{*}=\operatorname{sgn}\left(-\lambda^{2} e^{2 \mu u}\right)=$ -1 and $\varepsilon=\operatorname{sgn}\left(\lambda^{2}\left(1-\mu^{2}\right) e^{2 \mu u}\right)=1$ for $|\mu|<1$, and $\varepsilon=-1$ for $|\mu|>1$. Thus, the Vranceanu rotational surface is timelike pseudo-umbilical for $|\mu|<1$ and it is spacelike pseudo-umbilical with negative definite metric for $|\mu|>1$.

We omit the proof of the next theorem because it is similar to the proof of Theorem 4.4 . 
For $\overline{c_{0}} \neq 0$ and $\theta>0$ let us define the following functions

$$
\bar{\Phi}\left(\theta, b, \varepsilon, \varepsilon^{*}\right)=\int_{0}^{\theta} \sqrt{\frac{\varepsilon^{*}{\overline{c_{0}}}^{2}\left(\cosh ^{2} \eta-b^{2} \sinh ^{2} \eta\right)}{\varepsilon^{*}{\overline{c_{0}}}^{2}\left(\cosh ^{2} \eta-b^{2} \sinh ^{2} \eta\right)-\varepsilon}} d \eta
$$

and

$$
\bar{\Omega}\left(\theta, b, \varepsilon, \varepsilon^{*}\right)=\int_{0}^{\theta} \sqrt{\frac{\varepsilon^{*}{\overline{c_{0}}}^{2}\left(\sinh ^{2} \eta-b^{2} \cosh ^{2} \eta\right)}{\varepsilon^{*}{\overline{c_{0}}}^{2}\left(\sinh ^{2} \eta-b^{2} \cosh ^{2} \eta\right)+\varepsilon}} d \eta .
$$

such that the integrands are real valued functions.

Theorem 4.4. Let $M_{2}(b)$ be a non-planar rotational surface in the pseudoEuclidean space $\mathbb{E}_{2}^{4}$ given by (3.9). Then,

(a) $M_{2}(b)$ is a spacelike pseudo-umbilical surface in $\mathbb{E}_{2}^{4}$ if and only if the component functions of the unit speed profile curve $\beta$ of $M_{2}(b)$ are given by one of the followings:

$(a-1)$

$$
x(\theta)=\bar{c} e^{\psi(\theta)} \cosh \theta \text { and } z(\theta)=\bar{c} e^{\psi(\theta)} \sinh \theta,
$$

where $\psi(\theta)=\bar{\Phi}(\theta, b, 1,1), 0<b \leq 1$ and ${\overline{c_{0}}}^{2}\left(\cosh ^{2} \theta-b^{2} \sinh ^{2} \theta\right)>1$ for some $\overline{c_{0}} \in \mathbb{R}$ and $\bar{c} \in \mathbb{R}_{+}$;

$$
x(\theta)=\bar{c} e^{\psi(\theta)} \cosh \theta \text { and } z(\theta)=\bar{c} e^{\psi(\theta)} \sinh \theta,
$$

where $\psi(\theta)=\bar{\Phi}(\theta, b,-1,-1), b>1$ and $\bar{c} \in \mathbb{R}_{+}$. In this case, the surface $M_{2}(b)$ has negative definite metric;

$$
x(\theta)=\bar{c} e^{\varphi(\theta)} \sinh \theta \text { and } z(\theta)=\bar{c} e^{\varphi(\theta)} \cosh \theta,
$$

where $\varphi(\theta)=\bar{\Omega}(\theta, b, 1,1), 0<b<1$ and $\bar{c} \in \mathbb{R}_{+}$;

$(a-4)$

$$
x(\theta)=\bar{c} e^{\varphi(\theta)} \sinh \theta \text { and } z(\theta)=\bar{c} e^{\varphi(\theta)} \cosh \theta,
$$

where $\varphi(\theta)=\bar{\Omega}(\theta, b,-1,-1), b \geq 1$ and ${\overline{c_{0}}}^{2}\left(b^{2} \cosh ^{2} \theta-\sinh ^{2} \theta\right)>1$ for some $\overline{c_{0}} \in \mathbb{R}$ and $\bar{c} \in \mathbb{R}_{+}$. In this case, the surface $M_{2}(b)$ has negative definite metric;

$$
x(\theta)=r_{0} \sinh \theta \text { and } z(\theta)=r_{0} \cosh \theta,
$$

where $r_{0}$ is non-zero constant and $0<b<1$. In this case, the surface $M_{2}(b)$ lies in $\mathbb{H}_{1}^{3}\left(-r_{0}^{-2}\right) \subset \mathbb{E}_{2}^{4}$;

$(a-6)$

$$
x(\theta)=r_{0} \cosh \theta \text { and } z(\theta)=r_{0} \sinh \theta,
$$

where $r_{0}$ is non-zero constant and $b>1$. In this case, the surface $M_{2}(b)$ is lying in $\mathbb{S}_{2}^{3}\left(r_{0}^{-2}\right) \subset \mathbb{E}_{2}^{4}$ with negative definite metric. 
(b) $M_{2}(b)$ is a timelike pseudo-umbilical surface in $\mathbb{E}_{2}^{4}$ if and only if the component functions of the unit speed profile curve $\beta$ of $M_{2}(b)$ are given by one of the followings:

$(b-1)$

$$
x(\theta)=\bar{c} e^{\psi(\theta)} \cosh \theta \text { and } z(\theta)=\bar{c} e^{\psi(\theta)} \sinh \theta,
$$

where $\psi(\theta)=\bar{\Phi}(\theta, b, 1,-1), b>1$ and ${\overline{c_{0}}}^{2}\left(b^{2} \sinh ^{2} \theta-\cosh ^{2} \theta\right)>1$ for some $\overline{c_{0}} \in \mathbb{R}$ and $\bar{c} \in \mathbb{R}_{+}$;

$$
x(\theta)=\bar{c} e^{\psi(\theta)} \cosh \theta \text { and } z(\theta)=\bar{c} e^{\psi(\theta)} \sinh \theta,
$$

where $\psi(\theta)=\bar{\Phi}(\theta, b,-1,1), 0<b \leq 1$ and $\bar{c} \in \mathbb{R}_{+}$;

$$
x(\theta)=\bar{c} e^{\varphi(\theta)} \sinh \theta \text { and } z(\theta)=\bar{c} e^{\varphi(\theta)} \cosh \theta,
$$

where $\varphi(\theta)=\bar{\Omega}(\theta, b, 1,-1), b \geq 1$ and $\bar{c} \in \mathbb{R}_{+}$;

$(b-4)$

$$
x(\theta)=\bar{c} e^{\varphi(\theta)} \sinh \theta \text { and } z(\theta)=\bar{c} e^{\varphi(\theta)} \cosh \theta,
$$

where $\varphi(\theta)=\bar{\Omega}(\theta, b,-1,1), 0<b<1$ and ${\overline{c_{0}}}^{2}\left(\sinh ^{2} \theta-b^{2} \cosh ^{2} \theta\right)>1$ for some $\overline{c_{0}} \in \mathbb{R}$ and $\bar{c} \in \mathbb{R}_{+}$;

$$
x(\theta)=r_{0} \sinh \theta \text { and } z(\theta)=r_{0} \cosh \theta,
$$

where $r_{0}$ is non-zero constant and $b \geq 1$. In this case, the surface $M_{2}(b)$ lies in $\mathbb{H}_{1}^{3}\left(-r_{0}^{-2}\right) \subset \mathbb{E}_{2}^{4}$;

$$
x(\theta)=r_{0} \cosh \theta \text { and } z(\theta)=r_{0} \sinh \theta,
$$

where $r_{0}$ is non-zero constant and $0<b \leq 1$. In this case, the surface $M_{2}(b)$ lies in $\mathbb{S}_{2}^{3}\left(r_{0}^{-2}\right) \subset \mathbb{E}_{2}^{4}$.

5

$$
\begin{gathered}
\text { Pseudo-Umbilical Rotational } \\
\text { Surfaces with Pointwise 1-Type } \\
\text { Gauss Map }
\end{gathered}
$$

In this section, we determine pseudo-umbilical rotational surfaces in $\mathbb{E}_{2}^{4}$ with pointwise 1-type Gauss map of first kind and second kind.

Theorem 5.1. There exists no pseudo-umbilical rotational surface defined by (3.1) in $\mathbb{E}_{2}^{4}$ with pointwise 1-type Gauss map of the second kind. 
Proof. Assume that $M_{1}(b)$ is a non-planar regular pseudo-umbilical rotational surface in $\mathbb{E}_{2}^{4}$ defined by (3.1). From equation (2.7), the Laplacian of the Gauss map of the rotational surface $M_{1}(b)$ is given by

$$
\begin{aligned}
\Delta \nu= & \|h\|^{2} \nu+2 h_{12}^{4}\left(\varepsilon^{*} h_{22}^{3}-\varepsilon h_{11}^{3}\right) e_{1} \wedge e_{2} \\
& +\omega_{34}\left(e_{1}\right)\left(\varepsilon h_{11}^{3}+\varepsilon^{*} h_{22}^{3}\right) e_{1} \wedge e_{3}+\left(\varepsilon \varepsilon^{*} e_{2}\left(h_{11}^{3}\right)+e_{2}\left(h_{22}^{3}\right)\right) e_{2} \wedge e_{4} .
\end{aligned}
$$

Since $\varepsilon^{*} h_{11}^{3}=\varepsilon h_{22}^{3}$, equation (5.1) becomes

$$
\Delta \nu=\|h\|^{2} \nu+2 \varepsilon h_{11}^{3} \omega_{34}\left(e_{1}\right) e_{1} \wedge e_{3}+2 \varepsilon h_{12}^{4} \omega_{34}\left(e_{1}\right) e_{2} \wedge e_{4} .
$$

Suppose that $M_{1}(b)$ has pointwise 1-type Gauss map of second kind. Comparing (1.2) and (5.2), we get

$$
\begin{aligned}
f\left(1+\varepsilon \varepsilon^{*} C_{34}\right) & =\|h\|^{2}, \\
f C_{13} & =-2 \varepsilon^{*} h_{11}^{3} \omega_{34}\left(e_{1}\right), \\
f C_{24} & =-2 \varepsilon^{*} h_{12}^{4} \omega_{34}\left(e_{1}\right), \\
C_{12} & =C_{14}=C_{23}=0 .
\end{aligned}
$$

From (5.4) and (5.5), we have

$$
h_{12}^{4} C_{13}-h_{11}^{3} C_{24}=0 .
$$

When we write the equation (2.9) for $i=2$, we obtain

$$
h_{11}^{3} C_{13}-h_{12}^{4} C_{24}=0 .
$$

Since the Gauss map $\nu$ is of the second kind, equations (5.7) and (5.8) must have non-zero solution which implies $\left(h_{11}^{3}\right)^{2}-\left(h_{12}^{4}\right)^{2}=0$. Considering the first equations in (3.5) and (3.6) we have $\left(b^{2}-1\right)\left(b^{2} y^{2}(u) w^{\prime 2}(u)-w^{2}(u) y^{\prime 2}(u)\right)=0$. If $b^{2} y^{2}(u) w^{\prime 2}(u)-w^{2}(u) y^{\prime 2}(u)=0$, by solving this equation and $y^{\prime 2}(u)-w^{\prime 2}(u)=$ $\varepsilon A^{2}$ together, we get

$$
y^{\prime 2}(u)=-\varepsilon \varepsilon^{*} b^{2} \frac{A^{2}}{q^{2}} y^{2}(u) \text { and } w^{\prime 2}(u)=-\varepsilon \varepsilon^{*} \frac{A^{2}}{q^{2}} w^{2}(u)
$$

where $A=\sqrt{\varepsilon\left(y^{\prime 2}(u)-w^{\prime 2}(u)\right)} \neq 0$ and $q=\sqrt{\varepsilon^{*}\left(w^{2}(u)-b^{2} y^{2}(u)\right)} \neq 0$. Differentiating equations in (5.9) with respect to $u$, we obtain

$$
\begin{aligned}
2 y^{\prime}(u) y^{\prime \prime}(u) & =-\varepsilon \varepsilon^{*}\left(\frac{A^{2}}{q^{2}}\right)^{\prime} y^{2}(u)-2 \varepsilon \varepsilon^{*} b^{2} \frac{A^{2}}{q^{2}} y(u) y^{\prime}(u), \\
2 w^{\prime}(u) w^{\prime \prime}(u) & =-\varepsilon \varepsilon^{*}\left(\frac{A^{2}}{q^{2}}\right)^{\prime} w^{2}(u)-2 \varepsilon \varepsilon^{*} b^{2} \frac{A^{2}}{q^{2}} w(u) w^{\prime}(u) .
\end{aligned}
$$

If we multiply these equations by $-w^{\prime 2}(u)$ and $y^{\prime 2}(u)$, respectively, add them and also consider $b^{2} y^{2}(u){w^{\prime}}^{2}(u)-w^{2}(u) y^{\prime 2}(u)=0$, we get

$$
y^{\prime}(u) w^{\prime}(u)\left(y^{\prime}(u) w^{\prime \prime}(u)-w^{\prime}(u) y^{\prime \prime}(u)-\varepsilon \varepsilon^{*}\left(\frac{A^{2}}{q^{2}}\right)\left(y(u) w^{\prime}(u)-w(u) y^{\prime}(u)\right)\right)=0 .
$$


If $y=y_{0}=$ constant or $w=w_{0}=$ constant on an open subinterval of $I$, then $M_{1}(b)$ is a planar rotational surface. So, there is an open subinterval $J \subset I$ on which $y^{\prime}(u) w^{\prime}(u) \neq 0$, that is,

$$
y^{\prime}(u) w^{\prime \prime}(u)-w^{\prime}(u) y^{\prime \prime}(u)-\varepsilon \varepsilon^{*} \frac{A^{2}}{q^{2}}\left(b^{2} y(u) w^{\prime}(u)-w(u) y^{\prime}(u)\right)=0 .
$$

Using (3.5) in the equation given above, we get $\varepsilon \varepsilon^{*} h_{11}^{3}+h_{22}^{3}=0$. On the other hand, from the equation $(3.17), M_{1}(b)$ has zero mean curvature vector in $\mathbb{E}_{2}^{4}$. That is contradiction to the definition of pseudo-umbilical surface. Thus, $b^{2} y^{2}(u) w^{\prime 2}(u)-w^{2}(u) y^{\prime 2}(u) \neq 0$, that is, $b=1$. In this case, $h_{12}^{4}=-\varepsilon \varepsilon^{*} h_{11}^{3}$ and $\omega_{34}\left(e_{1}\right)=-\varepsilon \varepsilon^{*} \omega_{12}\left(e_{1}\right)$. Thus, from the equation (5.7) $C_{13}=-\varepsilon \varepsilon^{*} C_{24}$. Also, $C_{34}$ is zero due to equations (2.10) and (2.13) for $i=2$. On the other hand, from (2.11) for $i=1$ we have $\omega_{34}\left(e_{1}\right)=0$ which is a contradiction. Thus, $\nu$ is not of pointwise 1-type of second kind.

Note that if $\nu$ were pointwise 1-type of first kind, it would happen that from (5.4) and (5.5) $h_{11}^{3}=h_{12}^{4}=0$ or $\omega_{34}\left(e_{1}\right)=0$.

In the case $h_{11}^{3}=h_{12}^{4}=0, M_{1}(b)$ lies in the 3 -dimensional Euclidean or pseudo-Euclidean space. Thus, we omit this case.

For $\omega_{34}\left(e_{1}\right)=0$, we obtained some class of rotational surfaces as seen in the proof of Theorem 4.2. Thus, we conclude the following results:

Corollary 5.2. Let $M_{1}(b)$ be a non-planar pseudo-umbilical rotational surface in the pseudo-Euclidean space $\mathbb{E}_{2}^{4}$ given by (3.1). Then, $M_{1}$ has pointwise 1type Gauss map of the first kind if and only if the component functions of the unit speed profile curve $\alpha$ of $M_{1}(b)$ are given by one of the followings:

$i$.

$$
y(\theta)=r_{0} \sinh \theta \text { and } w(\theta)=r_{0} \cosh \theta,
$$

where $r_{0}$ is non-zero constant and $0<b \leq 1$. In this case, $M_{1}(b)$ is $a$ spacelike surface in $\mathbb{H}_{1}^{3}\left(-r_{0}^{-2}\right) \subset \mathbb{E}_{2}^{4}$;

ii.

$$
y(\theta)=r_{0} \cosh \theta \text { and } w(\theta)=r_{0} \sinh \theta,
$$

where $r_{0}$ is non-zero constant and $b \geq 1$. In this case, $M_{1}(b)$ is a spacalike surface with negative definite metric in $\mathbb{S}_{2}^{3}\left(r_{0}^{-2}\right) \subset \mathbb{E}_{2}^{4}$;

iii.

$$
y(\theta)=r_{0} \sinh \theta \text { and } w(\theta)=r_{0} \cosh \theta,
$$

where $r_{0}$ is non-zero constant and $b>1$. In this case, $M_{1}(b)$ is a timelike surface in $\mathbb{H}_{1}^{3}\left(-r_{0}^{-2}\right) \subset \mathbb{E}_{2}^{4}$;

$i v$.

$$
y(\theta)=r_{0} \cosh \theta \text { and } w(\theta)=r_{0} \sinh \theta,
$$

where $r_{0}$ is non-zero constant and $0<b<1$. In this case, $M_{1}(b)$ is a timelike surface in $\mathbb{S}_{2}^{3}\left(r_{0}^{-2}\right) \subset \mathbb{E}_{2}^{4}$. 
Similarly, we can give same results for the rotational surface $M_{2}(b)$ given by (3.9).

Theorem 5.3. There exists no pseudo-umbilical rotational surface defined by (3.9) in $\mathbb{E}_{2}^{4}$ with pointwise 1-type Gauss map of the second kind.

Corollary 5.4. Let $M_{2}(b)$ be a non-planar pseudo-umbilical rotational surface in the pseudo-Euclidean space $\mathbb{E}_{2}^{4}$ given by (3.9). Then, $M_{2}$ has pointwise 1type Gauss map of the first kind if and only if the component functions of the unit speed profile curve $\beta$ of $M_{2}(b)$ are given by one of the followings:

$i$.

$$
x(\theta)=r_{0} \sinh \theta \text { and } z(\theta)=r_{0} \cosh \theta,
$$

where $r_{0}$ is non-zero constant and $0<b<1$. In this case, $M_{2}(b)$ is a spacelike surface lying in $\mathbb{H}_{1}^{3}\left(-r_{0}^{-2}\right) \subset \mathbb{E}_{2}^{4}$;

ii.

$$
x(\theta)=r_{0} \cosh \theta \text { and } z(\theta)=r_{0} \sinh \theta,
$$

where $r_{0}$ is non-zero constant and $b>1$. In this case, $M_{2}(b)$ is a spacelike surface lying in $\mathbb{S}_{2}^{3}\left(r_{0}^{-2}\right) \subset \mathbb{E}_{2}^{4}$ with negative definite metric;

iii.

$$
x(\theta)=r_{0} \sinh \theta \text { and } z(\theta)=r_{0} \cosh \theta,
$$

where $r_{0}$ is non-zero constant and $b \geq 1$. In this case, $M_{2}(b)$ is a timelike surface lying in $\mathbb{H}_{1}^{3}\left(-r_{0}^{-2}\right) \subset \mathbb{E}_{2}^{4}$

$i v$.

$$
x(\theta)=r_{0} \cosh \theta \text { and } z(\theta)=r_{0} \sinh \theta,
$$

where $r_{0}$ is non-zero constant and $0<b \leq 1$. In this case, $M_{2}(b)$ is a timelike surface lying in $\mathbb{S}_{2}^{3}\left(r_{0}^{-2}\right) \subset \mathbb{E}_{2}^{4}$.

\section{REFERENCES}

[1] Aksoyak, F. K., Yayll, Y., General rotational surfaces with pointwise 1-type Gauss map in pseudo-Euclidean space $\mathbb{E}_{2}^{4}$, Indian J. Pure Appl. Math. 46 (2015), 107-118.

[2] Aleksieva, Y., Milousheva, V., Turgay, N. C., General rotational surfaces in pseudo-Euclidean 4-space with neutral metric, Bull. Malays. Math. Sci. Soc., (accepted, see DOI:10.1007/s40840-016-0425-0).

[3] Arslan, K., Milousheva, V., Meridian surfaces of elliptic or hyperbolic type with pointwise 1-type Gauss map in Minkowski 4-space, Taiwanese J. of Math. 20 (2016), 311-332. 
[4] Bektaş, B., Canfes, E. Ö., Dursun, U., On rotational surfaces in pseudoEuclidean space $\mathbb{E}_{t}^{4}$ with pointwise 1-type Gauss map, Acta Univ. Apulensis Math. Inform. 45 (2016), 43-59.

[5] Cao, X. F., Pseudo-umbilical submanifolds of constant curvature Riemannian manifolds, Glasgow Math. J. 43 (2001), 129-133.

[6] Chen, B.-Y., Pseudo-umbilical submanifolds of a Riemannian manifold of constant curvature II, J. Math. Soc. Japan 25 (1973), 105-114.

[7] Chen, B.-Y., Total mean curvature and submanifolds of finite type, World Scientific, Singapor-New Jersey-London, 1984.

[8] Chen, B.-Y., Piccinni, P., Submanifolds with finite type Gauss map, Bull. Austral. Mat. Soc. 35, (1987), 161-186.

[9] Chen, B.-Y., A report on submanifolds of finite type, Soochow J. Math. 22 (1996), 117-337.

[10] Chen, B.-Y., Total mean curvature and submanifolds of finite type, 2nd edition, World Scientific Publishing Co. Pte. Ltd., Hackensack, NJ, 2015.

[11] Choi, M., Yoon, D. W., Surfaces of revolution with pointwise 1-type Gauss map in pseudo-Galilean space, Bull. Korean Math. Soc. 53 (2016), 519530.

[12] Cole, F. N., On rotations in space of four dimensions, Amer. J. Math. 12 (1890), 191-210.

[13] Dursun, U., Hypersurfaces with pointwise 1-type Gauss map in LorentzMinkowski space, Proc. Est. Acad. Sci. 58, (2009), 146-161.

[14] Dursun, U., Turgay, N. C., Minimal and pseudo-umbilical rotational surfaces in Euclidean space $\mathbb{E}^{4}$, Mediterr. J. Math. 10, (2013), 497-506.

[15] HuiLi, L., GuiLi, L., Rotation surfaces with constant mean curvature in 4-dimensional pseudo-Euclidean space, Kyushu J. Math. 48, (1994), 35-42.

[16] Kim, Y. H., Yoon, D. W., Ruled surfaces with pointwise 1-type Gauss map, J. Geom. Phys. 34, (2000), 191-205.

[17] Kim, Y. H., Yoon, D. W., Classification of rotation surfaces in pseudoEuclidean space, J. Korean Math. Soc. 41 (2004), 379-396.

[18] Milousheva, V., Turgay, N. C., Quasi-minimal Lorentz surfaces with pointwise 1-type Gauss map in pseudo-Euclidean 4-space, J. Geometry Phys. 106, (2016), 171183.

[19] Moore, C. L. E., Surfaces of rotation in a space of four dimensions, Annals. of Math. 21 (1919), 81-93. 